\title{
Letalidad y sobrevida de pacientes con tuberculosis hospitalizados en el Instituto Nacional del Tórax
}

\author{
CRISTIÁN MEDINA A.*, FRANCISCO ARANCIBIA H.*, CRISTIÁN DEZA E.*, \\ PATRICIO VALLEJO V.*, IVÁN NAKOUZI S.* y CARLOS PEÑA M.**
}

\author{
Fatality rate and survival amongst discharged inpatients with tuberculosis, Instituto Nacional \\ del Torax, Chile
}

Background: Tuberculosis (TB) still is a public health issue in Chile. In the year 2015 TB incidence rate was 16 cases per 100.000 habitants and case fatality rate reached 10,8\% of patients belonging to the last TB treatment follow-up of pulmonary cases cohort (2013). It is well noted higher case fatality rate in TB hospitalized patients. The aim of this study is to determine our hospital case-fatality rate, recognize main risks factors involved and establish survival at 6 months since hospital discharge. Methods: We conducted an observational retrospective study in the Instituto Nacional del Tórax (INT) including inpatients adults over 15 years old between July 2012 and June 2015 whose diagnosis at discharge time was tuberculosis. Demographic data, comorbidities, laboratory, microbiological and radiological test, hospital stay, treatment, complications and mortality at 6 months were recorded. We used a logistic regression model to identify mortality-associated factors and to analyze the survival using Kaplan-Meier's method. Results: 100 patients whose average age was $46 \pm 19$ years-old were included. $64 \%$ were male. Pulmonary tuberculosis predominated (79 cases). The hospital stay was $27 \pm 32$ days. In-hospital case fatality rate was higher in pulmonary TB compared to other TB forms $111.4 \%$ and $8 \%$ respectively). Survival at 6 months was $91 \%$. Multivariate analysis showed that increased case fatality rate was associated with albumin $\leq 3.0 \mathrm{~g} / \mathrm{dL}$, thrombocytopenia, and shock. Conclusions: case fatality rate in our hospitalized TB patients is similar to that described in the country. We must pay attention in pulmonary TB inpatients, which have decreased levels of albumin and platelet, or develop septic shock.

Key words: Tuberculosis; Hospital Mortality; lethality; risk factors; public health.

\section{Resumen}

Introducción: La Tuberculosis (TBC) aún es un problema de salud pública en Chile. El 2015, la incidencia en todas las formas fue 16 casos $x 100.000$ habitantes, y en la cohorte de casos de TBC pulmonar adultos en tratamiento, la letalidad fue 10,8\% (2013). Un subgrupo de estos pacientes requieren hospitalización y tienen mayor morbimortalidad. Objetivo: Conocer la letalidad hospitalaria, los factores riesgo y determinar la sobrevida a 6 meses del diagnóstico e inicio del tratamiento. Método: Estudio retrospectivo, observacional que incluyó a todos los pacientes $>15$ años, hospitalizados en el Instituto Nacional del Tórax (INT) entre el 1 julio 2012 al 30 Junio 2015 y cuyo diagnóstico de egreso fue TBC. Se registraron datos demográficos, comorbilidades, exámenes de laboratorio, microbiológicos, radiológicos, estadía hospitalaria, tratamiento, complicaciones y letalidad a 6 meses. Para identificar factores asociados a letalidad, utilizamos un modelo de regresión logística y para sobrevida se utilizó la curva de Kaplan-Meier. Resultados: Se incluyeron 100 pacientes, edad $46 \pm 19$ años, 64\% varones. Predominó la TBC pulmonar (79\%). La estadía hospitalaria fue $27 \pm 32$ días. La letalidad intrahospitalaria fue 8\% en TBC y 11,4\% en TBC pulmonar. La sobrevida a los 6 meses fue $91 \%$. El análisis multivariado mostró que la letalidad se asoció estadísticamente con niveles de albúmina $\leq 3,0$ $\mathrm{g} / \mathrm{dl}$, trombocitopenia y shock séptico. Conclusiones: La letalidad en pacientes hospitalizados con TBC es similar a la descrita en el país. Se debe poner especial atención en pacientes con TBC pulmonar que tienen hipoalbuminemia, trombocitopenia al ingreso, o desarrollan shock séptico.

Palabras clave: Tuberculosis; Mortalidad Hospitalaria; Letalidad; Factores de riesgo; salud pública.

\footnotetext{
* Servicio de Medicina Respiratoria, Instituto Nacional del Tórax, Servicio de Salud Metropolitano Oriente. Chile.

** Hospital San Borja-Arriarán, Servicio de Salud Metropolitano Central. Chile.
} 


\section{Introducción}

La tuberculosis (TBC), continúa siendo un importante problema de salud pública en todo el mundo y una de las enfermedades transmisibles con mayor letalidad ${ }^{1}$. Se estiman, el año 2015, en 10,4 millones los casos nuevos de tuberculosis y en 1,8 millones $(17 \%)$ las muertes anuales ${ }^{2}$. Por otro lado se ha reportado, en EE.UU, un costo de los pacientes hospitalizados por tuberculosis de 2 billones de dólares anuales ${ }^{3}$.

Chile, desde la segunda mitad del siglo XX pertenece al selecto grupo de países con baja prevalencia de Tuberculosis de Latinoamérica y el Caribe. Actualmente su tasa de incidencia se encuentra por debajo del umbral de eliminación (menos de 20 casos nuevos por 100.000 habitantes/año). Sin embargo, la TBC aún está lejos de ser erradicada en nuestro país. En el año 2015 la incidencia nacional fue de 16 casos por 100.000 habitantes por año ${ }^{4}$. Por otro lado, en el año 2013, el porcentaje de fallecidos en los casos nuevos de tuberculosis pulmonar confirmados por bacteriología, en mayores de 15 años, alcanzó un $10,8 \%{ }^{5}$.

Una de las estrategias para reducir la mortalidad por $\mathrm{TBC}$, en pacientes hospitalizados, es realizar un manejo clínico más intensivo y estrategias de prevención, entre ellas, identificar factores que incrementan el riesgo de muerte ${ }^{5}$.

Si bien, los pacientes con tuberculosis pueden ser tratados de forma ambulatoria, un grupo significativo requiere hospitalización, tanto por motivos de proceso diagnóstico como para el manejo de las complicaciones ${ }^{4}$. En el año 2014, de un total de 2.383 casos de tuberculosis, el número de egresos hospitalarios informados por el Ministerio de Salud (MINSAL) fue de 1.409 (59,1\%) pacientes ${ }^{5,6}$. En nuestro país, se dispone de escasa información sobre la evolución y el desenlace de los pacientes hospitalizados por esta enfermedad.

Los objetivos de nuestro estudio fueron: conocer la letalidad hospitalaria en pacientes con tuberculosis tratados en el Instituto Nacional del Tórax (INT), determinar los factores pronósticos de la letalidad durante el tratamiento y la sobrevida a 6 meses de iniciado el tratamiento.

\section{Material y Métodos}

Estudio retrospectivo observacional en el cual fueron incluidos todos los pacientes mayores de 15 años, hospitalizados en el INT durante el período comprendido entre el 1 de julio de 2012 y 30 de junio de 2015. Se registraron datos demográficos, comorbilidades, nivel de escolaridad, procedencia, comienzo de los síntomas, síntomas, hallazgos relevantes del examen físico, exámenes de laboratorio de ingreso, método diagnóstico de $\mathrm{TBC}$, datos radiológicos, tratamiento, estadía hospitalaria, complicaciones y mortalidad intrahospitalaria y a los 6 meses de egreso. Se consideró positivo el consumo de alcohol cuando el paciente refería una ingesta de alcohol de $1 \mathrm{o}$ más veces por semana.

Los datos fueron obtenidos de la ficha clínica de paciente. Para conocer su condición, a los 6 meses se obtuvo información del Registro Civil. Este estudio fue aprobado por el Comité de éticacientífico del Servicio de Salud Metropolitano Oriente.

El diagnóstico de tuberculosis se confirmó por alguno de los siguientes métodos: (1) cultivo de M. tuberculosis positivo, (2) resultado de PCR para M. tuberculosis positivo, (3) baciloscopia positiva, (4) hallazgos sugerentes de infección por tuberculosis en la histología, y/o (5) manifestaciones clínico-radiológicas típicas de tuberculosis en ausencia de diagnóstico alternativo. Los pacientes con diagnóstico de tuberculosis fueron todos tratados de acuerdo a las normas nacionales vigentes del programa de control de la tuberculosis del Ministerio de Salud ${ }^{7,8}$ y recibieron los fármacos en forma supervisada por técnicos paramédicos capacitados. Los estudios de sensibilidad mediante el uso de biología molecular de muestras de expectoración y líquidos corporales, fueron realizados con la técnica de geneXpert MTB/RIF. Todas las muestras para estudio de sensibilidad fueron confirmadas por el Instituto de Salud Pública. La baciloscopia y cultivo de Koch se realizó mediante tinción de Ziehl-Nielsen y cultivos en medios líquidos.

\section{Análisis estadístico}

Los resultados son expresados como: valores promedio \pm desviación estándar para variables en escala numérica y en porcentajes para medidas registradas en escala nominal. Las variables continuas fueron comparadas utilizando una prueba " $t$ " de student. Para las variables categóricas se utilizó la prueba de $\chi^{2}$ o prueba de Fisher. Para identificar factores asociados con mayor letalidad en pacientes con TBC, usamos un modelo de regresión logística con varibles categorizadas y con el método de selección por pasos hacia adelante. La variables fueron incluidas en el análisis multivariado cuando estas tenían un nivel de significancia estadística en el analisis univariado $(\mathrm{p}<0,05)$. Las siguientes variable fueron incluidas en el modelo: edad $>40$ años, nivel de albúmina $\leq 3,0 \mathrm{~g} / \mathrm{dl}$, plaquetas $<150.000$ 
por $\mathrm{mm}^{3}$, consumo de alcohol, diabetes mellitus, insuficiencia renal aguda, shock y requerimiento de soporte respiratorio mecánico (ventilación no invasiva y/o ventilación mecánica). Los resultados del análisis multivariado son reportados como odds ratios (OR) e intervalos de confianza (IC) para el 95\%. Para todas las pruebas, el nivel de significación se estableció en 0,05 y con dos colas. Todos los pacientes fueron incluidos en el analisis univariado y multivariado.

Para analizar la sobrevida se utilizó la curva de Kaplan-Meier. Todos los datos fueron procesados con el programa estadístico IBM SPSS Statistic, versión 22.

\section{Resultados}

Durante los 36 meses del período en estudio, fueron incluidos un total de 100 pacientes que tuvieron diagnóstico de tuberculosis al momento de su egreso hospitalario. Fueron excluidos dos pacientes por información insuficiente y un caso, que correspondió a un paciente extranjero que no aceptó recibir tratamiento antituberculoso.

\section{Características clínicas}

La edad promedio de los pacientes fue de $46 \pm$ 19 DS (rango 15-91) años, el 64\% eran de género masculino y el $13 \%$ extranjeros.

El hábito tabáquico estaba presente en el $22 \%$ de los casos y el consumo de alcohol en el $24 \%$. Las comorbilidades más frecuentes reportadas fueron: diabetes mellitus en el $23 \%$, patología pulmonar obstructiva crónica en el $15 \%$ e infección por VIH en el 11\%. El 18\% de los casos tenía antecedentes previos de tuberculosis. Otras características generales de la población estudiada, son presentadas en la Tabla 1.

Los síntomas de presentación más frecuentes fueron: tos en el $76 \%$ de los casos, y baja de peso en el 52\%. La hemoptisis se presentó en el $15 \%$ de ellos. El 48\% de los casos, tenían el antecedente de síntomas de más de 90 días de duración.

En la radiografía de tórax, el $30 \%$ de los pacientes presentaban compromiso bilateral, las lesiones cavitadas se observaron en el $41 \%$ y la presencia de derrame pleural en el $31 \%$.

En los exámenes de laboratorio destaca que la hemoglobina fue de 12,1 $\pm 2,3$ (rango 6,9-19) g/ $\mathrm{dL}$; recuento plaquetas 361.857 (79.000-946.000) por $\mathrm{mm}^{3}$; la VHS $65,6 \pm 36 \mathrm{~mm} / \mathrm{h}$; la albúmina plasmática 3,2 $\pm 0,7$ (rango $1,4-4,4$ ) g/dL y el ADA (adenosina deaminasa) en el líquido pleu$\mathrm{ral}=38,3$ (rango 12-60) UI/L.

Al ingreso hospitalario, solo el $30 \%$ de los pacientes tenía diagnóstico de TBC pulmonar ya formulado. Al egreso hospitalario, el $79 \%$ de pacientes correspondió a tuberculosis pulmonar y el $21 \%$ a tuberculosis extrapulmonar (Tabla 2).

La estadía hospitalaria fue de $27 \pm 32$ días, sin observar diferencias significativas entre los pacientes que sobrevivieron y aquellos fallecidos.

Tabla 1. Características generales de los pacientes con tuberculosis

\begin{tabular}{|c|c|}
\hline Característica & Valor \\
\hline Número de pacientes & 100 \\
\hline Edad, Promedio \pm DS (años) & $46 \pm 19$ \\
\hline Género masculino & $64 \%$ \\
\hline Extranjeros & $13 \%$ \\
\hline Antecedentes de Tuberculosis previa & $18 \%$ \\
\hline $\begin{array}{l}\text { Comorbilidad pulmonar } \\
\text { Secuelas tuberculosis } \\
\text { Asma } \\
\text { EPD* } \\
\text { Otros }\end{array}$ & $\begin{array}{r}15 \% \\
4 \% \\
3 \% \\
3 \% \\
5 \%\end{array}$ \\
\hline Diabetes mellitus & $23 \%$ \\
\hline Infección por $\mathrm{VIH}^{* *}$ & $11 \%$ \\
\hline $\begin{array}{l}\text { Hábitos } \\
\text { Consumo de alcohol } \\
\text { Tabaquismo actual } \\
\text { Consumo marihuana } \\
\text { Consumo cocaína }\end{array}$ & $\begin{array}{r}24 \% \\
22 \% \\
11 \% \\
3 \%\end{array}$ \\
\hline $\begin{array}{l}\text { Escolaridad } \\
\text { Analfabeto } \\
\text { Enseñanza básica } \\
\text { Enseñanza media } \\
\text { Enseñanza universitaria }\end{array}$ & $\begin{array}{r}3 \% \\
49 \% \\
38 \% \\
9 \%\end{array}$ \\
\hline Estadía hospitalaria, Promedio \pm DS (días) & $27 \pm 32$ \\
\hline Letalidad hospitalaria & $8 \%$ \\
\hline Letalidad 6 meses & $9 \%$ \\
\hline
\end{tabular}

*EPD, enfermedad pulmonar difusa; **VIH, virus de inmunodeficiencia humana.

Tabla 2. Clasificación diagnóstica de los pacientes con Tuberculosis $(n=100)$

\begin{tabular}{|lc|}
\hline $\begin{array}{l}\text { Formas de presentación } \\
\text { de la tuberculosis }\end{array}$ & (\%) \\
\hline Tuberculosis pulmonar & 79 \\
Pleuropulmonar & 14 \\
Tuberculosis extrapulmonar & 21 \\
Pleural & 15 \\
Ganglionar & 3 \\
Articular & 1 \\
Mal de Pott & 1 \\
Urogenital & 1 \\
\hline
\end{tabular}




\section{Complicaciones}

El 17\% de los pacientes presentó complicaciones que fueron las siguientes: shock séptico en 8 casos, falla respiratoria aguda en 6 , de los cuales todos requirieron ventilación no invasiva y 4 ventilación mecánica invasiva. Cinco pacientes presentaron insuficiencia renal aguda, empiema 7 , e insuficiencia hepática un paciente.

\section{Letalidad}

Durante los 6 meses de tratamiento, se registró un total de 9 fallecidos, 8 de los cuales fallecieron durante la hospitalización, siendo en todos ellos la TBC la causa de muerte. Un caso falleció post-alta por un infarto agudo al miocardio. La letalidad intrahospitalaria por TBC fue de $8 \%$ (IC 95\%: 3-14,1) y de un 11,4\% en el subgrupo de TBC pulmonar. La sobrevida a los 6 meses fue $91 \%$. (IC 95\%: 85,9-97) La Figura 1, muestra la curva de sobrevida de pacientes con tuberculosis hospitalizados.

\section{Análisis univariado}

En el análisis univariado para evaluar los factores pronósticos de mortalidad, se encontró que: edad $>40$ años, nivel de albúmina $\leq 3,0 \mathrm{~g} / \mathrm{dl}$, plaquetas $<150.000$ por $\mathrm{mm}^{3}$, consumo de alcohol, diabetes mellitus, insuficiencia renal aguda, shock séptico y requerimiento de soporte respiratorio mecánico, resultaron estar significativamente asociados (Tabla 3 ).

\section{Análisis multivariado}

El análisis de regresión logística muestra que la hipoalbuminemia (OR: 7,38; 95\% IC, $1,66-32,65 ; \mathrm{p}=0,008)$ y la plaquetopenia (OR: 14,56; 95\% IC: $1,29-163,8 ; p=0,030)$, medidas al ingreso de la hospitalización, y la presencia de shock séptico (OR: 86,35; 95\% IC, 6,16-

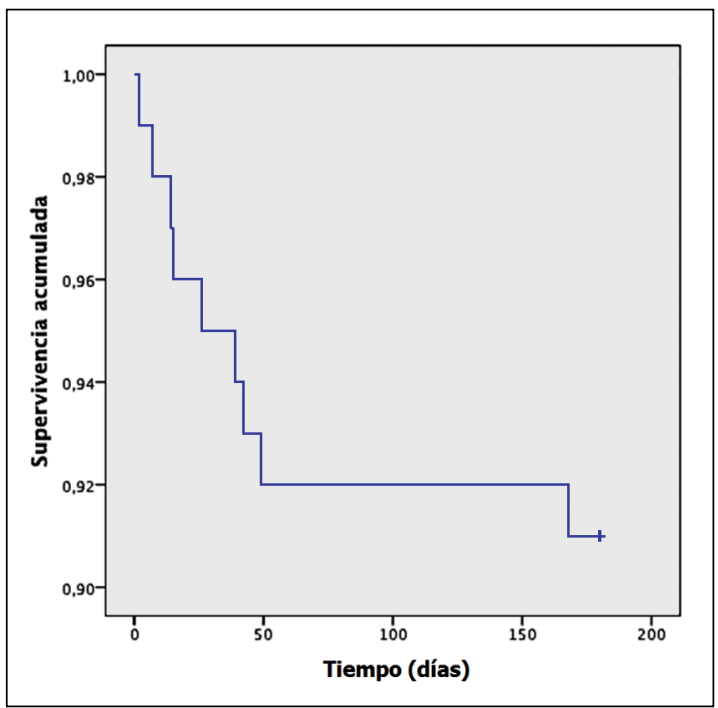

Figura 1. Curva de sobrevida de Kaplan-Meier de pacientes hospitalizados con tuberculosis.

1.210,28; $\mathrm{p}=0,001)$ durante la hospitalización, resultaron factores pronósticos independientes de mortalidad hospitalaria en pacientes con TBC.

\section{Discusión}

Entre los principales hallazgos en este estudio destacamos: (1) la letalidad hospitalaria de los pacientes con TBC fue de $8 \%$, con una sobrevida del $91 \%$ a los 6 meses y (2) la hipoalbuminemia, la trombocitopenia al ingreso y el shock séptico durante la hospitalización, son factores de riesgo independientes de mortalidad. La letalidad por TBC en nuestro estudio, es algo mayor a lo encontrado por Hansel y cols en EE.UU., $4,9 \%{ }^{9}$

Tabla 3. Análisis univariado de factores pronósticos de mortalidad en pacientes hospitalizados por tuberculosis

\begin{tabular}{|lccccc|}
\hline Variables & $\begin{array}{c}\text { Vivos } \\
(\mathbf{n}=\mathbf{9 1})\end{array}$ & $\begin{array}{c}\text { Fallecidos } \\
(\mathbf{n}=\mathbf{9 )}\end{array}$ & \multicolumn{2}{c|}{ OR $(\mathbf{9 5 \%}$ IC) } & p \\
\hline Edad $>40$ años & $49 / 91(54 \%)$ & $8 / 9(89 \%)$ & 10,0 & $(1,24-81,15)$ & 0,01 \\
\hline Albúmina $\leq 3,0 \mathrm{~g} / \mathrm{dL}$ & $23 / 81(28 \%)$ & $8 / 9(89 \%)$ & 20,17 & $(2,39-170,48)$ & $<0,0001$ \\
Plaquetas $<150.000$ & $3 / 89(3,4 \%)$ & $4 / 9(44 \%)$ & 22,933 & $(3,99-131,65)$ & $<0,0001$ \\
Diabetes mellitus & $18 / 91(20 \%)$ & $5 / 9(56 \%)$ & 5,069 & $(1,24-20,81)$ & 0,015 \\
Consumo de alcohol & $19 / 91(21 \%)$ & $5 / 9(56 \%)$ & 4,737 & $(1,16-19,38)$ & 0,020 \\
Insuficiencia renal aguda & $2 / 87(2,3 \%)$ & $3 / 9(33 \%)$ & 21,25 & $(2,96-152,59)$ & $<0,0001$ \\
Soporte ventilatorio & $2 / 91(2,2 \%)$ & $6 / 9(67 \%)$ & 89 & $(12,4-638,79)$ & $<0,0001$ \\
\hline Shock & $1 / 87(1,1 \%)$ & $7 / 9(78 \%)$ & 301 & $(24,19-3,744,89)$ & $<0,0001$ \\
\hline
\end{tabular}

Datos son porcentajes y proporciones. 
y por Pepper y cols ${ }^{10}$ en Sudáfrica, $5,6 \%$. Por el contrario, es menor a lo reportado por Lubart y cols $^{11}$ en Israel, $13 \%$; Silva y cols ${ }^{12}$ en Brasil, $17 \%$; Chuquiyauri y col ${ }^{13}$ en Perú, $18,4 \%$; y Rao y cols $^{14}$ en EE.UU., $28,1 \%$, en pacientes con TBC hospitalizados (Tabla 4). Esta diferencia en la letalidad, puede ser explicada por varios factores, entre ellos, se debe considerar el hecho que el INT, es un centro nacional de derivación, lo que puede condicionar la selección de casos de mayor severidad o complejidad. Otros factores de importancia son las comorbilidades presentes en las poblaciones estudiadas. En el estudio israelí, el $40 \%$ de los fallecidos tenían insuficiencia cardiaca isquémica ${ }^{11}$. En cambio, en el estudio realizado en Brasil ${ }^{12}$ y Perú ${ }^{13}$, los pacientes fallecidos presentaban co-infección por VIH en el $63 \%$ y $66 \%$, respectivamente. La tuberculosis es la causa más común de muerte en pacientes que presentan co-infección por VIH en países en desarrollo ${ }^{15}$. No obstante, esto ha mejorado con la incorporación en la última década de la terapia antiretroviral. Abdool-Karim y cols, encontraron que la iniciación de terapia antiretroviral durante la terapia antituberculosa, disminuye la mortalidad en $56 \%{ }^{16}$. En nuestro estudio, solo 1 de los 11 pacientes que tenía co-infección por VIH falleció y había suspendido la terapia antiretroviral meses antes. En cambio, los otros pacientes estaban en tratamiento antiretroviral regular.

La sobrevida a los 6 meses de iniciado el tratamiento antituberculoso fue de $91 \%$, sin embargo, la mayoría de los pacientes que fallecieron, lo hicieron por tuberculosis pulmonar durante la hospitalización. En ellos, las comorbilidades más frecuentes asociadas fueron la diabetes mellitus $(56 \%)$ y el consumo de alcohol (56\%). Solo un caso falleció después de la hospitalización por una causa diferente a la tuberculosis.

En el estudio de Hansen y cols. ${ }^{9}$, se encontró que aquellos pacientes con un elevado índice de comorbilidad de Charlson ${ }^{17}$ (método pronóstico de mortalidad según el número de comorbilidades seleccionadas), tenían un mayor riesgo de mortalidad hospitalaria, $\mathrm{OR}=1,57$.

A pesar de que nuestros pacientes recibieron la mejor terapia antituberculosa disponible de acuerdo a las normas nacionales del programa de control de la tuberculosis ${ }^{7,8}$, un $8 \%$ de ellos falleció. Esta cifra se acerca a lo reportado por el Ministerio de Salud, el año 2013, de un 10,8\% de mortalidad global ${ }^{5}$. Dado la gravedad y complicaciones que tiene estos pacientes, como se muestra en nuestro estudio, es muy probable que la mayoría de los pacientes que fallecen por tuberculosis en Chile corresponda a pacientes hospitalizados.

En nuestro estudio, encontramos que la hipoalbuminemia, es un factor de mal pronóstico. Esto ha sido comunicado previamente por Lubart y cols ${ }^{11}$, que encontró en el análisis multivariado que la hipoalbuminemia era uno de los más importantes factores relacionados con mortalidad en pacientes hospitalizados con TBC. En otro estudio, Tang y cols ${ }^{18}$ encontraron que la hipoalbuminemia es factor pronóstico independiente de pobre respuesta en pacientes con tuberculosis resistente a multidrogas (MDR). Otros factores

Tabla 4. Estudios que analizan la letalidad y los factores de riesgo de mortalidad de pacientes con tuberculosis hospitalizados

\begin{tabular}{|c|c|c|c|c|}
\hline Autor & Año & $\mathbf{n}$ & Letalidad, n (\%) & *Factores riesgo mortalidad \\
\hline $\mathrm{Rao}^{14}$ & 1998 & 203 & $57(28)$ & $\begin{array}{l}\text { Falla respiratoria, edad }<60 \text { años, Insuficiencia renal } \\
\text { terminal, malnutrición, inmunosupresión, disnea }\end{array}$ \\
\hline Chuquiyauri ${ }^{13}$ & 2004 & 1248 & $230(18)$ & Edad > 30 años, co-infección con VIH \\
\hline Hansel $^{9}$ & 2004 & 2.279 & $112(4,9)$ & $\begin{array}{l}\text { Edad, comorbilidad, admisión por departamento de } \\
\text { emergencias }\end{array}$ \\
\hline Erbes $^{25, \S}$ & 2006 & 58 & $15(26)$ & $\begin{array}{l}\text { Falla renal aguda, ventilación mecánica, pancreatitis } \\
\text { crónica, sepsis, SDRA, neumonía nosocomial. }\end{array}$ \\
\hline Lubart $^{11}$ & 2007 & 461 & $65(13)$ & $\begin{array}{l}\text { Enfermedad cardiaca isquémica, edad mayor, ca- } \\
\text { quexia, corticoides, hipoalbuminemia }\end{array}$ \\
\hline Silva $^{12}$ & 2010 & 311 & $50(16)$ & Necesidad de ventilación mecánica \\
\hline Getahun $^{19}$ & 2011 & 6.450 & $252(3,7)$ & $\begin{array}{l}\text { Edad, peso }<35 \mathrm{~kg} \text {, categoría del paciente, año de } \\
\text { enrolamiento, centro de salud de tratamiento }\end{array}$ \\
\hline
\end{tabular}

*Análisis multivariado, con modelo de regresión logística o regresión de Cox. \$Pacientes hospitalizados en UCI. SDRA: Síndrome distrés respiratorio agudo; VIH: virus de inmunodeficiencia humana. 
de riesgo relacionados a mortalidad son malnutrición, bajo peso e índice de masa corporal bajo $^{11,14,18,19}$. Esto es muy importante, y sugiere la conveniencia de evaluar el estado nutricional del paciente desde el inicio del tratamiento e iniciar intervenciones con los suplementos nutricionales necesarios, de forma precoz.

Con respecto a la trombocitopenia es conocido que es un marcador de mal pronóstico en neumonía y sepsis ${ }^{20}$. En tuberculosis, la trombocitopenia es infrecuente y está usualmente asociada con TBC diseminada o TBC miliar ${ }^{21}$. En la etiopatogenia de la trombocitopenia de estos pacientes se encuentra: infiltración de la médula ósea en TBC diseminada, coagulación intravascular diseminada relacionado a TBC, generación de anticuerpos anti plaquetarios inducidos por rifampicina y el púrpura trombótico trombocitopénico (PTT) ${ }^{21,22}$. La trombocitopenia severa como complicación de la tuberculosis es poco común, pero puede ser potencialmente letal ${ }^{23}$. No obstante, puede representar un fenómeno reversible si el diagnóstico es precoz y el tratamiento adecuado.

Los pacientes con TBC que requieren hospitalización en la unidad de paciente crítico tienen una elevada letalidad ${ }^{24}$, siendo las complicaciones como el shock séptico y la falla respiratoria aguda las causas muerte. En el estudio de Rollas $\mathrm{y} \operatorname{cols}^{24}$, la principal causa de muerte fue shock séptico en 5 de los 7 pacientes fallecidos. Así también, Erbes y cols ${ }^{25}$ estudiaron a 58 pacientes con tuberculosis hospitalizados en la UCI con una letalidad del $26 \%$, estando la sepsis presente en el $25,8 \%$ de los casos. En nuestro estudio, 6 de los 8 pacientes que fallecieron durante la hospitalización y que requirieron atención en la Unidad de Paciente Crítico presentaron shock séptico. En la Tabla 4 se presentan varios estudios que analizan los factores de riesgo de mortalidad de pacientes hospitalizados con tuberculosis.

Finalmente, en otros estudios las variables: género masculino ${ }^{26} \mathrm{y}$ la edad, sobre todo mayores de 65 años $^{26,27}$, son asociados con mortalidad por tuberculosis. En nuestro estudio estas variables no fueron retenidas en el modelo multivariado. Es posible que influya el tamaño muestral que es menor a los estudios antes mencionados.

No obstante que Chile tiene una baja incidencia de TBC comparada con la mayoría de los países de Latinoamérica y el Caribe, aún estamos lejos de su erradicación y de convertirnos así en un país de baja incidencia ${ }^{28}$. Es más, se ha observado en los últimos años una detención en la velocidad de reducción de la tasa de incidencia y una mortalidad preocupantemente elevada para una enfermedad prevenible y tratable 5 .

\section{Conclusiones}

Nuestro estudio muestra una letalidad por TBC, en pacientes hospitalizados, de un $8 \%$ y una sobrevida a los 6 meses de 91\%. La mayoría falleció por causa de la TBC pulmonar. En el análisis multivariado, la hipoalbuminemia, la trombocitopenia, y el shock séptico fueron factores pronóstico de mortalidad.

De acuerdo a nuestros resultados, debemos redoblar los esfuerzos para lograr un diagnóstico más precoz de la tuberculosis, instaurar la terapia antituberculosa, optimizar el diagnostico y manejo de las comorbilidades y realizar una evaluación nutricional al inicio del tratamiento, de tal forma de reducir la letalidad. Es posible que los resultados de nuestro estudio, por haberse realizado en un solo centro de salud y de referencia nacional, puedan no ser extrapolable a otros hospitales.

\section{Bibliografía}

1.- CENTERS FOR DISEASE CONTROL AND PREVENTION. Data and statistics on tuberculosis. Available at: http://www.cdc.gov/TB/statistics/ (Consultado el 30 de septiembre de 2015).

2.- WORLD HEALTH ORGANIZATION. Global Tuberculosis Report 2016, http:/www.who.int/tb/publications/ global_report/en/ (Consultado el 30 de diciembre de 2016).

3.- MIRSAEIDIA M, ALLENB M B, EBRAHIMIA G, AND SCHRAUFNAGELA D. Hospital costs in the US for pulmonary mycobacterial diseases. Int J Mycobacteriol 2015; 4: 217-21.

4.- WORLD HEALTH ORGANIZATION. TB burden estimates and country-reported TB data. https://extranet. who.int $/$ sree $/$ Reports?op=Replet\&name $=\% 2 \mathrm{FWHO}$ HQ_Reports\%2FG2\%2FPROD\%2FEXT\%2FTBCo untryProfile \&ISO $2=\mathrm{CL} \& \mathrm{LAN}=\mathrm{ES} \&$ outtype $=\mathrm{html}$. (Consultado el 13 de octubre de 2016).

5.- MINISTERIO DE SALUD DE CHILE (MINSAL). Tuberculosis, informe de situación actual, año 2014. http:// web.minsal.cl/sites/default/files/Informe_tbc_2014.pdf, (Consultado el 15 de septiembre de 2015).

6.- MINISTERIO DE SALUD. Egresos Hospitalarios. Departamento de estadísticas e información de salud. http://intradeis.minsal.cl/egresoshospitalarios/menu_publica_nueva/menu_publica_nueva.htm. (Consultado el 29 de Diciembre de 2016).

7.- MINISTERIO DE SALUD. Manual de Organización y Normas Técnicas. Programa Nacional de Control de la Tuberculosis. Santiago. Chile. 2005. (Consultado el 15 de septiembre de 2015).

8.- MINISTERIO DE SALUD. Normas Técnicas Para el Control y la Eliminación de la Tuberculosis Santiago 
de Chile-2014. http://web.minsal.cl/sites/default/files/ NORMA TECNICA TUBERCULOSIS.pdf. (Consultado el 15 septiembre de 2015).

9.- HANSEL N N, MERRIMAN B, HAPONIK E F, DIETTE G B. Hospitalizations for tuberculosis in the United States in 2000: predictors of in-hospital mortality. Chest 2004; 126: 1079-86.

10.- PEPPER D J, SCHOMAKER M, WILKINSON R J, DE AZEVEDO V, AND MAARTENS G. Independent predictors of tuberculosis mortality in a high HIV prevalence setting: a retrospective cohort study. AIDS Res Ther 2015; 12: 35 .

11.- LUBART E, LIDGI M, LEIBOVITZ A, RABINOVITZ C, SEGAL R. Mortality of Patients Hospitalized for Active Tuberculosis in Israel. IMAJ 2007; 9: 870-3.

12.- SILVA D R, MENEGOTTO D M, SCHULZ L F, GAZZANA M B, DALCIN P T R. Clinical characteristics and evolution of non-HIV-infected immunocompromised patients with an in-hospital diagnosis of tuberculosis. J Bras Pneumol 2010; 36: 475-84.

13.- CHUQUIYAURI R, VERDONCK K, GONZÁLES E, ZAMUDIO E, ECHEVARRIA J, SEAS C, et al. Morbimortalidad de pacientes con tuberculosis hospitalizados en el Departamento de enfermedades infecciosas, tropicales y dermatológicas del Hospital Nacional Cayetano Heredia, Lima - Perú entre los años 1990 y 2000. Rev Med Hered [online] 2004; 15 :203-10.

14.- RAO V K, IADEMARCO E P, FRASER V J, KOLLEF M H. The Impact of Comorbidity on Mortality Following In-hospital Diagnosis of Tuberculosis. Chest 1998; 114: 1244- 52.

15- SMALL P M, FUJIWARA P I. Management of tuberculosis in the United States. N Engl J Med 2001; 345: 189-200.

16.- ABDOOL KARIM S S, NAIDOO K, GROBLER A, PADAYATCHI N, BAXTER C, GRAY A, et al. Timing of initiation of antiretroviral drugs during tuberculosis therapy. N Engl J Med 2010; 362: 697-706.

17.- CHARLSON M E, POMPEI P, ALES K L, MACKENZIE C R. A new method of classifying prognostic comorbidity in longitudinal studies: development and validation. J Chronic Dis 1987; 40: 373-83.

18.- TANG S, TAN S, YAO L, LI F, LI L, GUO X, et al. Risk factors for poor treatment outcomes in patients with MDR-TB and XDR-TB in China: retrospective multi-center investigation. PLoS One 2013; 8: e82943.
19.- GETAHUN B, AMENI G, BIADGILIGN S, MEDHIN G. Mortality and associated risk factors in a cohort of tuberculosis patients treated under DOTS programme in Addis Abeba, Ethiopia. BMC Infect Dis 2011; 16; 11: 127.

20.- MANDELL L A, WUNDERINK R G, ANZUETO A, BARTLETT J G, CAMPBELL G D, DEAN N C, et al; Infectious Diseases Society of America; American Thoracic Society . Infectious Diseases Society of America/ American Thoracic Society consensus guidelines on the management of community acquired pneumonia in adults. Clin Infect Dis 2007; 44 (suppl 2): S27-S72 .

21.- RUDRAJIT P. State of the Globe: Immune Thrombocytopenia, an Uncommon Complication in Tuberculosis. J Glob Infect Dis 2014; 6:93-4.

22.- DAGAONKAR R S, UDWADIA Z F. Disseminated tuberculosis with immune thrombocytopenic purpura. Lung India 2012; 29: 63-5.

23.- BOOTS R J, ROBERTS A W, MCEVOY D. Immune thrombocytopenia complicating pulmonary tuberculosis: case report and investigation of mechanisms. Thorax 1992; 47: 396-7.

24.- ROLLAS K, KARA A, ORTAÇ ERSOY N E, ÖZMEN SÜNER K, GÜLLÜ MN, ÖCAL S, et al. Acute tuberculosis in the intensive care unit. Turk J Med Sci 2015; 45: 882-7.

25.- ERBES R, OETTEL K, RAFFENBERG M, MAUCH H, SCHMIDT-IOANAS M, LODE H. Characteristics and outcome of patients with active pulmonary tuberculosis requiring intensive care. Eur Respir J 2006; 27 : 1223-8.

26.- DALE K, TAY E, TREVAN P, DENHOLM J T. Mortality among tuberculosis cases in Victoria, 2002-2013: case fatality and factors associated with death. Int $\mathrm{J}$ Tuberc Lung Dis 2016; 20: 515-23.

27.- HEUNIS J C, KIGOZI N G, CHIKOBVU P, BOTHA S, VAN RENSBURG HD. Risk factors for mortality in TB patients: a 10-year electronic record review in a South African province. BMC Public Health 2017; 17: 38.

28.- Organización Panamericana de la Salud. La tuberculosis en la Región de las Américas: Informe Regional 2012. Epidemiología, control y financiamiento. Washington, DC: OPS, 2013. http://www.paho.org /hq/index.php?option $=$ com_docman\&task $=$ doc view\&gid=22954\&Itemid=270. (Consultado el 29 de diciembre de 2016).

Correspondencia a:

Dr. Francisco Arancibia

Instituto Nacional del Tórax,

Avda. José M. Infante 717, Providencia.

Santiago de Chile

Email: fearancibia@gmail.com 